\title{
A Case Series of Recurrent Viral RNA Positivity in Recovered COVID-19 Chinese Patients
}

\author{
Kenneth I. Zheng, M.D. ' , Xiao-Bo Wang, M.D. ${ }^{2}$, Xiang-Hong Jin, M.D. ${ }^{3}$, \\ Wen-Yue Liu, M.D. ${ }^{4}$, Feng Gao, M.D. ${ }^{5}$, Yong-Ping Chen, M.D. ${ }^{1,6,7}$, and Ming-Hua Zheng, \\ M.D., Ph.D. ${ }^{1,6,7}$
}

${ }^{1}$ NAFLD Research Center, Department of Hepatology, The First Affiliated Hospital of Wenzhou Medical University, Wenzhou, China; ${ }^{2}$ Department of Critical Care Medicine, Wenzhou Central Hospital, Wenzhou, China; ${ }^{3}$ Department of Haematology, The First Affiliated Hospital of Wenzhou Medical University, Wenzhou, China; ${ }^{4}$ Department of Endocrinology, The First Affiliated Hospital of Wenzhou Medical University, Wenzhou, China; ${ }^{5}$ Department of Gastroenterology, The First Affiliated Hospital of Wenzhou Medical University, Wenzhou, China; ${ }^{6}$ nstitute of Hepatology, Wenzhou Medical University, Wenzhou, China; ${ }^{7}$ Key Laboratory of Diagnosis and Treatment for The Development of Chronic Liver Disease in Zhejiang Province, Wenzhou, China.

\author{
Abbreviations \\ SARS- Severe acute respiratory syndrome-associated \\ CoV-2 coronavirus 2 \\ COVID-19 Coronavirus disease 2019 \\ CT Computed tomography \\ RT-PCR Real-time reverse transcriptase-polymerase chain \\ reaction \\ J Gen Intern Med 35(7):2205-6 \\ DOI: $10.1007 / \mathrm{s} 11606-020-05822-1$ \\ (c) Society of General Internal Medicine 2020
}

\section{INTRODUCTION}

The outbreak of coronavirus disease 2019 (COVID-19) has been recently declared a pandemic by the World Health Organization. ${ }^{1}$ Cases of positive real-time reverse transcriptasepolymerase chain reaction (RT-PCR) results in patients recovered from coronavirus disease 2019 (COVID-19) have been recently reported. ${ }^{2}$ However, little is currently known about the prevalence of recurrent positive RT-PCR test results in these patients.

\section{METHODS}

We examined twenty patients successfully treated for COVID19 who had a 2-week post-discharge follow-up period who were admitted to the First Affiliated Hospital of Wenzhou Medical University and Wenzhou Central Hospital in Wenzhou, China, between January 25, 2020, and February 26, 2020. Of note, Wenzhou City was the second epicenter of the SARS-CoV-2 outbreak and had the second largest number of cases reported. ${ }^{3}$

Discharge criteria were as follows: $(1)>3$ days of normal body temperature, (2) resolved respiratory symptoms, (3) substantially improved acute exudative lesions on chest computed tomography (CT), and (4) negative RT-PCR test results for both two consecutive salivary tests (with at least 1 day between tests) and one fecal nucleic acid (RNA) test. ${ }^{4}$ In all these

Published online April 20, 2020 patients, treatment included nebulized $\alpha$-interferon, as recommended by the Chinese COVID-19 Management Guidance, ${ }^{5}$ until the aforementioned criteria for hospital discharge were met. Discharged patients were asked to follow strict protocols for self-isolation at designated hotels and away from family or relatives for 2 weeks. This study was approved by our local IRB, waiving informed consent for de-identified data.

\section{RESULTS}

At baseline, all SARS-CoV-2 infection-confirmed patients (14 men, 6 women) with age ranging from 23 to 57 years had fever, while 9 also had cough. Their white blood cell (WBC) and lymphocyte counts, expressed as mean $\pm \mathrm{SD}$, were $5.02 \pm 1.47 \times 10^{9} / \mathrm{L}$ and $1.31 \pm 0.49 \times 10^{9} / \mathrm{L}$, respectively. Their chest CT scans showed ground-glass opacities and consolidation that affected varying numbers of lobes with or without bilateral pulmonary involvement.

Seven days after hospital discharge, 3 patients who initially tested negative at discharge developed positive RT-PCR test results. One tested positive by fecal RNA, while two tested positive by both salivary and fecal RNA tests at 1 week of follow-up. Interestingly, all 20 patients returning for the week 2 follow-up tested negative for SARS-CoV-2, irrespective of sampling route. All three cases had improved with no increase in their temperature, and improvement in WBC and lymphocyte counts, as well as their CT scans. Compared with 17 patients with negative RT-PCR results at week 1 follow-up, there were no significant differences in WBC (6.0 vs. 4.9, $p=$ 0.38 ) or lymphocyte counts (1.9 vs. 1.6, $p=0.25$ ).

\section{DISCUSSION}

We found that 3 of 20 patients, 7 days after hospital discharge with negative RT-PCR tests, had a positive RT-PCR test. Two weeks after discharge, all three patients tested negative for viral RNA. Interestingly, there was no difference in symptoms 
between those who remained negative and those who were positive; all cases experienced steady improvement. Because all discharged patients followed a strict protocol for self-isolation, we believe that the RNA positivity at follow-up is unlikely to be due to reinfection. It remains also uncertain whether recovered COVID-19 patients with positive RT-PCR results are able to transmit the infection to others by close contact. These findings suggest that strict self-isolation protocols and extended follow-up periods might be needed for recovered COVID-19 patients.

Our case series, although the first observational study to longitudinally examine COVID-19 patients, has several important limitations. First, this is a small sample $(n=20)$, meaning that our results are preliminary and cannot be used to predict the proportion with recurrently positive tests. Secondly, while adherence to self-quarantine in our population is high, it is possible that this represents reinfection rather than persistent infection. Larger and longer-term prospective studies are needed to better understand the outcome of COVID-19.

Acknowledgments: The authors thank Prof. Christopher D. Byrne (Faculty of Medicine, University of Southampton and Southampton NIHR Biomedical Research Centre, University Hospital Southampton, UK) and Prof. Giovanni Targher (Department of Medicine, University and Azienda Ospedaliera Universitaria Integrata of Verona, Verona, Italy) for proof reading and comments on the manuscript.
Corresponding Author: Ming-Hua Zheng, M.D., Ph.D.; NAFLD Research Center, Department of Hepatology, The First Affiliated Hospital of Wenzhou Medical University, Wenzhou, China (e-mail: zhengmh@wmu.edu.cn).

Compliance with Ethical Standards:

This study was approved by the local ethics review board from the First Affiliated Hospital of Wenzhou Medical University and Wenzhou Central Hospital, waiving patient written informed consent for deidentified data.

Conflict of Interest: The authors declare that they do not have a conflict of interest.

\section{REFERENCES}

1. WHO characterizes COVID-19 as a pandemic. Published March 13, 2020. Accessed March 18, 2020. https://www.who.int/emergencies/diseases/ novel-coronavirus- 2019/events-as-they-happen.

2. Lan L, Xu D, Ye G, Xu D, Ye G, et al. Positive RT-PCR Test Results in Patients Recovered From COVID- 19. Jama 2020. https://doi.org/10. $1001 /$ jama.2020.2783.

3. Gong $\mathbf{F}$, Xiong $\mathbf{Y}$, Xiao J, Lin $\mathbf{L}$, Liu X, Wang $\mathbf{D}$, et al. China's local governments are combating COVID-19 with unprecedented responses from a Wenzhou governance perspective. Front Med. 2020. https://doi. org/10.1007/s11684-020-0755-z.

4. Huang $\mathbf{C}$, Wang $\mathbf{Y}, \mathbf{L i} \mathbf{X}$, et al. Clinical features of patients infected with 2019 novel coronavirus in Wuhan, China. Lancet 2020; 395(10223): 497506.

5. China National Health Commission. Diagnosis and treatment of COVID-19 in China (6th Edition). In Chinese. Published February 19, 2020. Accessed March 8, 2020. http://www.nhc.gov.cn/yzygj/s7653p/202002/ 8334a8326dd94d329df351d7da8aefc2.shtml.

Publisher's Note: Springer Nature remains neutral with regard to jurisdictional claims in published maps and institutional affiliations. 